PROCEEDINGS OF THE

AMERICAN MATHEMATICAL SOCIETY

Volume 140, Number 7, July 2012, Pages 2391-2399

S 0002-9939(2011)11088-8

Article electronically published on November 7, 2011

\title{
A MEASURE-THEORETIC VERSION OF THE DRAGOMIR-JENSEN INEQUALITY
}

\author{
J. M. ALDAZ
}

(Communicated by Edward C. Waymire)

\begin{abstract}
We extend Dragomir's refinement of Jensen's inequality from the discrete to the general case, identifying the equality conditions.
\end{abstract}

\section{INTRODUCTION}

Suppose we have a probability space $(\Omega, \mathcal{A}, P)$, an integrable function $X: \Omega \rightarrow \mathbb{R}$, and a real valued strictly convex function $\phi$ defined in some interval containing the range of $X$. By Jensen's inequality,

$$
E \phi(X)-\phi(E X) \geq 0
$$

with equality precisely when $X(\omega) \equiv E(X)$ for $P$-a.e. $\omega$. Thus, the left hand side of (11) provides a measure of the spread of $X$ around its mean value. Of course, in the important special case $\phi(t)=t^{2}$, the left hand side of (11) is just the variance of $X$. It is natural to study how these generalized variances change when the probability $P$ varies. The case of discrete probability measures with finite support was considered by S. S. Dragomir in Dra].

Here we note that Dragomir's clever proof (refining Jensen's inequality by using Jensen's inequality itself) can be used to extend his result to general probability spaces. Additionally, we identify the cases of equality when $\phi$ is strictly convex and present some immediate consequences.

\section{The Dragomir-Jensen inequality}

To motivate the inequality, consider the following simple example: Let $X$ and $Y$ be random variables on $(\Omega, \mathcal{A}, P)$, with densities $f_{X}$ and $f_{Y}$ respectively, such that the quotient $f_{Y} / f_{X}$ is well defined almost everywhere, or in other words, such that the distribution of $\mathrm{Y}$, defined by the push-forward (or induced) probability $Y_{*} P(A):=P(Y \in A)$, is absolutely continuous with respect to $X_{*} P(A):=$ $P(X \in A)$. If both $X$ and $Y$ have mean zero, then it is easy to bound the variance of $Y$ in terms of the variance of $X$ : Since

$$
\operatorname{Var}(Y)=\int_{-\infty}^{\infty} y^{2} f_{Y}(y) d y=\int_{-\infty}^{\infty} y^{2} \frac{f_{Y}(y)}{f_{X}(y)} f_{X}(y) d y
$$

Received by the editors December 31, 2010 and, in revised form, February 16, 2011 and February 18, 2011.

2010 Mathematics Subject Classification. Primary 26D15, 60A10.

Key words and phrases. Self-improvement, Jensen's inequality.

This work was partially supported by Grant MTM2009-12740-C03-03 of the D.G.I. of Spain.

(c)2011 American Mathematical Society 2391

Reverts to public domain 28 years from publication 
and $\operatorname{Var}(X)=\int_{-\infty}^{\infty} y^{2} f_{X}(y) d y$, replacing $\frac{f_{Y}(y)}{f_{X}(y)}$ in (2) by its essential supremum and by its essential infimum, we get

$$
\text { ess inf }\left(\frac{f_{Y}(y)}{f_{X}(y)}\right) \operatorname{Var}(X) \leq \operatorname{Var}(Y) \leq\left\|\frac{f_{Y}(y)}{f_{X}(y)}\right\|_{\infty} \operatorname{Var}(X) .
$$

In fact, (3) holds whenever the expected values of $X$ and $Y$ are finite, and not just for $\phi(t)=t^{2}$, but for arbitrary convex functions. This is the content of the (two sided) Dragomir-Jensen inequality (cf. Theorem 2.1 and Corollary 2.9), which generalizes (3) much in the same way as Jensen's inequality generalizes $\operatorname{Var}(X) \geq 0$.

Next we recall some basic notation and facts. Given an absolutely continuous measure $P \ll Q$, as usual, $\frac{d P}{d Q}$ denotes the Radon-Nikodym derivative of $P$ with respect to $Q$, and $\left\|\frac{d P}{d Q}\right\|_{\infty}$ denotes its essential supremum (which could be infinite). We adopt the standard measure-theoretic convention $\infty \cdot 0=0$ (recall that under any other convention, the monotone convergence theorem would fail). Of course, integrals are used here in the Lebesgue sense. In particular, to have $\int X d P$ well defined, it is assumed that either $\int X^{+} d P<\infty$ or $\int X^{-} d P<\infty$, where $X^{+}$and $X^{-}$respectively denote the positive and negative parts of $X$. Thus, we do not consider principal values.

Since we will be dealing with positive measures and, in fact, probabilities, by taking any representative of the Radon-Nikodym derivative and redefining it (if needed) on a set of measure zero, we may assume that $0 \leq \frac{d P}{d Q}<\infty$ (we use the same notation for the Radon-Nikodym derivative and its representative).

Theorem 2.1. Let $(\Omega, \mathcal{A})$ be a measurable space, let $P$ and $Q$ be probability measures defined on $(\Omega, \mathcal{A})$ such that $P \ll Q$, let $X: \Omega \rightarrow \mathbb{R}$ be integrable both with respect to $P$ and $Q$, and let the real valued function $\phi$ be convex in some (not necessarily bounded) interval containing the range of $X$. Then

$$
\int_{\Omega} \phi(X) d P-\phi\left(\int_{\Omega} X d P\right) \leq\left\|\frac{d P}{d Q}\right\|_{\infty}\left(\int_{\Omega} \phi(X) d Q-\phi\left(\int_{\Omega} X d Q\right)\right) .
$$

Regarding the equality conditions, to avoid trivialities we suppose that $P \neq Q$, and then we distinguish three cases.

1) Both sides of (4) take the value $\infty$ if and only if $\int_{\Omega} \phi(X) d P=\infty$, and then either $\int_{\Omega} \phi(X) d Q=\infty$ or $\left\|\frac{d P}{d Q}\right\|_{\infty}=\infty$.

Next, assume that $\phi$ is strictly convex and let $A:=\left\{\omega \in \Omega: \frac{d P}{d Q}(\omega)=\left\|\frac{d P}{d Q}\right\|_{\infty}\right\}$.

2) Both sides of (44) take the value 0 if and only if $X$ is $Q$-a.e. constant.

3) Both sides of (4) take the same value $a$, with $0<a<\infty$, if and only if the following three conditions hold:

a) $\int_{\Omega} \phi(X) d Q<\infty$ and $\left\|\frac{d P}{d Q}\right\|_{\infty}<\infty$.

b) There exists a constant $c$ such that $X \equiv c, Q$-a.e. on $\Omega \backslash A$, but $X$ is not $Q$-a.e. constant on $\Omega$.

c) $Q(A)>0, P(A)>0$, and $c=\int_{\Omega} X d Q=\frac{1}{Q(A)} \int_{A} X d Q=\int_{\Omega} X d P=$ $\frac{1}{P(A)} \int_{A} X d P$.

Example 2.2. Given $(\Omega, \mathcal{A}, P), A \subset X$ with $P(A)>0$, and $X: \Omega \rightarrow \mathbb{R}$ integrable, it is intuitively obvious (and not difficult to prove directly) that the variance $\operatorname{Var}_{A}(X)$ of $X$ restricted to $A$, with respect to the conditional probability 
$P_{A}(B):=P(B \mid A)$, cannot be much larger than the original variance of $X$ on all $\Omega$, with respect to $P$. This is now a special case of the preceding theorem: Since $\left\|\frac{d P_{A}}{d P}\right\|_{\infty}=\frac{1}{P(A)}$, setting $\phi(t)=t^{2}$ in (4) we get

$$
\operatorname{Var}_{A}(X) \leq \frac{\operatorname{Var}(X)}{P(A)}
$$

Example 2.3. Given $X: \Omega \rightarrow \mathbb{R}$ and $g: \mathbb{R} \rightarrow \mathbb{R}$, suppose we know the distribution of $X$ but there is some uncertainty about the value of one or several parameters, and we want to estimate how the variance of $g(X)$ is affected by this uncertainty. The preceding theorem can be used to this end.

Assume, for instance, that $X \sim N(0, \sigma)$, with $0<a \leq \sigma \leq b$. The zero mean assumption is made so the resulting expressions are simple, but the case where there is uncertainty both about the mean and the variance can be treated in the same way. Call $\operatorname{Var}_{1}(g(X))$ and $\operatorname{Var}_{2}(g(X))$ the variances obtained by supposing that $X$ has standard deviations $\sigma_{1}$ and $\sigma_{2}$ respectively, with $a \leq \sigma_{1} \leq \sigma_{2} \leq b$, and let $P$ and $Q$ be the corresponding laws for $X$. Then

and hence

$$
\frac{d P}{d Q}=\frac{\sigma_{2}}{\sigma_{1}} e^{\frac{x^{2}\left(\sigma_{1}-\sigma_{2}\right)}{2 \sigma_{1}^{2} \sigma_{2}^{2}}}, \quad \text { so } \quad\left\|\frac{d P}{d Q}\right\|_{\infty}=\frac{\sigma_{2}}{\sigma_{1}} \leq \frac{b}{a},
$$

$$
\operatorname{Var}_{1}(g(X)) \leq \frac{b}{a} \operatorname{Var}_{2}(g(X)) .
$$

If we additionally know that $g$ is compactly supported, we can present a simultaneous lower bound (in this regard, see also Corollary 2.9 below). Suppose, for simplicity, that the support of $g$ is contained in $[c, d]$, with $0<c<d$. Then

$$
\frac{d Q}{d P}=\frac{\sigma_{1}}{\sigma_{2}} e^{\frac{x^{2}\left(\sigma_{2}-\sigma_{1}\right)}{2 \sigma_{1}^{2} \sigma_{2}^{2}}} \leq \frac{\sigma_{1}}{\sigma_{2}} e^{\frac{d^{2}\left(\sigma_{2}-\sigma_{1}\right)}{2 \sigma_{1}^{2} \sigma_{2}^{2}}}, \quad \text { so } \quad \operatorname{Var}_{2}(g(X)) \leq \frac{\sigma_{1}}{\sigma_{2}} e^{\frac{d^{2}\left(\sigma_{2}-\sigma_{1}\right)}{2 \sigma_{1}^{2} \sigma_{2}^{2}}} \operatorname{Var}_{1}(g(X)),
$$

and hence

$$
\frac{\sigma_{2}}{\sigma_{1}} e^{\frac{d^{2}\left(\sigma_{1}-\sigma_{2}\right)}{2 \sigma_{1}^{2} \sigma_{2}^{2}}} \operatorname{Var}_{2}(g(X)) \leq \operatorname{Var}_{1}(g(X)) .
$$

Remark 2.4. It is natural to ask whether the hypothesis $P \ll Q$ in the theorem must be imposed on all of $\Omega$ or is it sufficient to consider just the set $\{X \neq 0\}$. To see that this is not enough, let $\Omega=[0,1]$, let $\mathcal{A}$ be the Lebesgue sets, and let $d P=d x$. Take $X=\chi_{[1 / 2,1]}$ and $d Q:=2 \chi_{[1 / 2,1]} d x$. Then $\frac{d P}{d Q}=\frac{1}{2}$ on $\{X \neq 0\}$, so restricted to $\{X \neq 0\}, P \ll Q$ and $\frac{d P}{d Q}$ is bounded. Let $\phi(x)=x^{2}$. Since $X$ is constant a.e. with respect to $Q$, the right hand side of (4) is zero, while the left hand side is just $1 / 2-1 / 4$.

Even if we extend $\frac{d P}{d Q}$ to all $[0,1]$ by setting $\frac{d P}{d Q}=\infty$ on $(0,1 / 2)$, the $\infty \times 0=0$ convention entails that (4) does not extend to pairs $P, Q$ when $P$ has a singular part.

Remark 2.5. The equality conditions, for the original Dragomir's result, where established in $\mathrm{Al}$. In the more general measure-theoretic setting, dealing only with bounded functions would be too restrictive, so the possibility that some term equals $\infty$ must be considered. Related to this issue is the fact that, since we are working on a probability space, the $L^{p}$ norm of any function is monotone increasing in $p$. It is thus natural to ask whether the Dragomir-Jensen inequality can be improved by replacing $\left\|\frac{d P}{d Q}\right\|_{\infty}$ with $c_{p}\left\|\frac{d P}{d Q}\right\|_{p}$ for some $p<\infty$ and some constant $c_{p}>0$. We 
show that $p=\infty$ is optimal. Fix $p<\infty$ with $p \geq 1$. Let $Q$ be Lebesgue measure on $(0,1]$ and let $\phi(x)=x^{2}$. Set $X(t)=t^{-1 / 2+1 /(4 p)}$ and $d P(t):=C t^{-1 /(2 p)} d t$, where the constant $C$ is chosen so $C t^{-1 /(2 p)}$ is a density. Then $X \in L^{1}(P) \cap L^{1}(Q)$, $\begin{aligned} \int_{\Omega} \phi(X) d Q<\infty, \int_{\Omega} \phi(X) d P & =\infty, \text { and } c_{p}\left\|\frac{d P}{d Q}\right\|_{p}<\infty, \text { so } \\ \int_{\Omega} \phi(X) d P-\phi\left(\int_{\Omega} X d P\right) & =\infty>c_{p}\left\|\frac{d P}{d Q}\right\|_{p}\left(\int_{\Omega} \phi(X) d Q-\phi\left(\int_{\Omega} X d Q\right)\right) .\end{aligned}$

On the other hand, $\left\|\frac{d P}{d Q}\right\|_{\infty}=\infty$ and $0<\int_{\Omega} \phi(X) d Q-\phi\left(\int_{\Omega} X d Q\right)$, so this is an instance where both sides of the Dragomir-Jensen inequality take the value $\infty$.

To make the structure of the proof of the theorem more transparent, we place some measure-theoretic details into two technical lemmas.

Lemma 2.6. Let $(\Omega, \mathcal{A})$ be a measurable space, let $P$ and $Q$ be probability measures defined on $(\Omega, \mathcal{A})$ such that $P \ll Q$, let $\frac{d P}{d Q}$ be essentially bounded, and let

$$
A:=\left\{\omega \in \Omega: \frac{d P}{d Q}(\omega)=\left\|\frac{d P}{d Q}\right\|_{\infty}\right\} .
$$

Then for every measurable set $B \subset A, P(B)=0$ if and only if $Q(B)=0$. In particular, $P(A)=0$ if and only if $Q(A)=0$.

Proof. Since both $P$ and $Q$ are probabilities, $\left\|\frac{d P}{d Q}\right\|_{\infty} \geq 1$. However, restricted to $A, P$ is just $\left\|\frac{d P}{d Q}\right\|_{\infty} Q$, so the result follows.

Lemma 2.7. With the notation and under the assumptions of the preceding lemma, let the measurable space $(\tilde{\Omega}, \tilde{\mathcal{A}})$ be the enlargement of $(\Omega, \mathcal{A})$ obtained by adding a new point $y$ to $\Omega$ and declaring it to be measurable. That is, $y \notin \Omega, \tilde{\Omega}:=\Omega \cup\{y\}$ and $\tilde{\mathcal{A}}:=\mathcal{A} \cup\{B \cup\{y\}: B \in \mathcal{A}\}$. Denote by $\delta_{y}$ the Dirac point mass at $y$. Then the set function $\tilde{P}$, defined on $(\tilde{\Omega}, \tilde{\mathcal{A}})$ by

$$
\tilde{P}(B):=Q(B \cap \Omega)-\left\|\frac{d P}{d Q}\right\|_{\infty}^{-1} P(B \cap \Omega)+\left\|\frac{d P}{d Q}\right\|_{\infty}^{-1} \delta_{y}(B),
$$

is a probability. Furthermore, $\tilde{P}(A)=0$, and for every measurable set $B \subset \Omega \backslash A$, $\tilde{P}(B)=0$ if and only if $Q(B)=0$.

Proof. Select any $w \in \Omega$, identify $\Omega$ with $\Omega \times\{0\}$, and set $y:=w \times\{1\}$ (this ensures that $y$ is actually a new point). Then let $\tilde{\Omega}:=\Omega \cup\{y\}$, and let $\tilde{\mathcal{A}}:=\mathcal{A} \cup\{B \cup\{y\}$ : $B \in \mathcal{A}\}$. Since $\tilde{\mathcal{A}}$ contains $\emptyset$, is closed under complementation, and also under countable unions, it is a $\sigma$-algebra. Additionally, $\{y\}$ is measurable, for $\emptyset \in \mathcal{A}$, whence $\{y\}=\emptyset \cup\{y\} \in \tilde{\mathcal{A}}$.

Let us check that $\tilde{P}$ is a probability. Clearly $\tilde{P}(\tilde{\Omega})=1$. To see that $\tilde{P}$ is nonnegative, we use the change of variable formula $\int_{\Omega} X d P=\int_{\Omega} X \frac{d P}{d Q} d Q$, and we conclude that for every $B \in \tilde{\mathcal{A}}$, we have

$$
\tilde{P}(B) \geq \int_{B \cap \Omega}\left(1-\left\|\frac{d P}{d Q}\right\|_{\infty}^{-1} \frac{d P}{d Q}\right) d Q \geq 0 .
$$


Note next that whenever $B \subset \Omega$ is measurable, (5) reduces to

$$
\tilde{P}(B)=Q(B)-\left\|\frac{d P}{d Q}\right\|_{\infty}^{-1} P(B) \leq Q(B) .
$$

In particular, $A:=\left\{w \in \Omega: \frac{d P}{d Q}(w)=\left\|\frac{d P}{d Q}\right\|_{\infty}\right\}$ has $\tilde{P}$-measure zero, since by (6),

$$
\tilde{P}(A):=Q(A)-\left\|\frac{d P}{d Q}\right\|_{\infty}^{-1} P(A)=Q(A)-\int_{A}\left\|\frac{d P}{d Q}\right\|_{\infty}^{-1} \frac{d P}{d Q} d Q=Q(A)-Q(A)=0 .
$$

Finally, to see that for any measurable $B \subset \Omega \backslash A, \tilde{P}(B)=0$ if and only if $Q(B)=0$, observe that if $Q(B)=0$, by (6) we have $\tilde{P}(B) \leq Q(B)=0$, while if $Q(B)>0$, since $1-\left\|\frac{d P}{d Q}\right\|_{\infty}^{-1} \frac{d P}{d Q}>0$ on $\Omega \backslash A$, we have

$$
\tilde{P}(B)=\int_{B}\left(1-\left\|\frac{d P}{d Q}\right\|_{\infty}^{-1} \frac{d P}{d Q}\right) d Q>0 .
$$

Proof of Theorem 2.1. Note that if $\frac{d P}{d Q}$ is unbounded (i.e., if $\left\|\frac{d P}{d Q}\right\|_{\infty}=\infty$ ), then the right hand side of (4) is $\infty$ unless $\int_{\Omega} \phi(X) d Q=\phi\left(\int_{\Omega} X d Q\right)$, in which case its value is zero (by standard convention). Hence, for (4) to hold we need to have $\int_{\Omega} \phi(X) d P=\phi\left(\int_{\Omega} X d P\right)$. This would be trivial if $\phi$ were strictly convex, for then $X$ would be constant $Q$-a.e., and thus also constant $P$-a.e. since $P \ll Q$. However, for $\phi$ not strictly convex, equality may occur for non-constant functions, and so a different argument is needed. What we do is to assume first that $\left\|\frac{d P}{d Q}\right\|_{\infty}<\infty$ and then handle the unbounded case via an approximation argument. Let us also suppose that both $\int_{\Omega} \phi(X) d Q<\infty$ and $\int_{\Omega} \phi(X) d P<\infty$. Then inequality (44) is equivalent to

$$
\phi\left(\int_{\Omega} X d Q\right) \leq \int_{\Omega} \phi(X) d Q-\left\|\frac{d P}{d Q}\right\|_{\infty}^{-1} \int_{\Omega} \phi(X) d P+\left\|\frac{d P}{d Q}\right\|_{\infty}^{-1} \phi\left(\int_{\Omega} X d P\right)
$$

simply by rearranging terms. However, (7) immediately follows from the usual Jensen's inequality, applied to the probability measure space $(\tilde{\Omega}, \tilde{\mathcal{A}}, \tilde{P})$ defined in Lemma 2.7, and to a suitable extension of $X$, given by $\tilde{X}:=X$ on $\Omega$, and $\tilde{X}(y):=$ $\int_{\Omega} X d P$. The function $\tilde{X}: \tilde{\Omega} \rightarrow \mathbb{R}$ is clearly $\tilde{\mathcal{A}}$ measurable. Furthermore,

$$
\begin{aligned}
\int_{\tilde{\Omega}} \tilde{X} d \tilde{P}= & \int_{\Omega} \tilde{X} d \tilde{P}+\int_{\{y\}} \tilde{X} d \tilde{P}=\int_{\Omega} X d Q-\left\|\frac{d P}{d Q}\right\|_{\infty}^{-1} \int_{\Omega} X d P \\
& +\left\|\frac{d P}{d Q}\right\|_{\infty}^{-1} \int_{\{y\}}\left(\int_{\Omega} X d P\right) d \delta_{y} \\
= & \int_{\Omega} X d Q
\end{aligned}
$$

so by Jensen's inequality on $(\tilde{\Omega}, \tilde{\mathcal{A}}, \tilde{P})$,

$$
\begin{gathered}
\phi\left(\int_{\Omega} X d Q\right)=\phi\left(\int_{\tilde{\Omega}} \tilde{X} d \tilde{P}\right) \leq \int_{\tilde{\Omega}} \phi(\tilde{X}) d \tilde{P} \\
=\int_{\Omega} \phi(X) d Q-\left\|\frac{d P}{d Q}\right\|_{\infty}^{-1} \int_{\Omega} \phi(X) d P+\left\|\frac{d P}{d Q}\right\|_{\infty}^{-1} \int_{\{y\}} \phi(\tilde{X}) d \delta_{y} .
\end{gathered}
$$


It follows from $\tilde{X}(y)=\int_{\Omega} X d P$ that $\int_{\{y\}} \phi(\tilde{X}) d \delta_{y}=\phi(\tilde{X}(y))=\phi\left(\int_{\Omega} X d P\right)$, so (7) holds and hence so does (4) when every term appearing there is finite. Suppose next that $\left\|\frac{d P}{d Q}\right\|_{\infty}=\infty$ and that $\int_{\Omega} \phi(X) d Q=\phi\left(\int_{\Omega} X d Q\right)$ (for otherwise (4) is trivial). Let us emphasize that we do not a priori assume that $\int_{\Omega} \phi(X) d P<\infty$; this will follow once $\int_{\Omega} \phi(X) d P=\phi\left(\int_{\Omega} X d P\right)$ is proven. Note however that $\int_{\Omega}(\phi(X))^{-} d P<\infty$, since $\int_{\Omega} \phi(X) d P \geq \phi\left(\int_{\Omega} X d P\right)>-\infty$. Define $d P_{n}:=\min \left\{\frac{d P}{d Q}, n\right\} d Q$ and observe that by the monotone convergence theorem, applied to $\min \left\{\frac{d P}{d Q}, n\right\}$, and separately to $X^{+} \min \left\{\frac{d P}{d Q}, n\right\}, X^{-} \min \left\{\frac{d P}{d Q}, n\right\}$, $(\phi(X))^{+} \min \left\{\frac{d P}{d Q}, n\right\}$, and $(\phi(X))^{-} \min \left\{\frac{d P}{d Q}, n\right\}$, we have

$$
\begin{gathered}
\lim _{n} P_{n}(\Omega)=\lim _{n} \int_{\Omega} \min \left\{\frac{d P}{d Q}, n\right\} d Q=\int_{\Omega} \frac{d P}{d Q} d Q=P(\Omega)=1, \\
\lim _{n} \frac{1}{P_{n}(\Omega)} \int_{\Omega} X \min \left\{\frac{d P}{d Q}, n\right\} d Q=\int_{\Omega} X \frac{d P}{d Q} d Q=\int_{\Omega} X d P,
\end{gathered}
$$

and

$$
\lim _{n} \frac{1}{P_{n}(\Omega)} \int_{\Omega} \phi(X) d P_{n}=\int_{\Omega} \phi(X) d P
$$

Since $\left\|\frac{d P_{n}}{d Q}\right\|_{\infty}=n$, we know from Jensen's inequality and the previous case that

$$
0 \leq \int_{\Omega} \phi(X) \frac{d P_{n}}{P_{n}(\Omega)}-\phi\left(\int_{\Omega} X \frac{d P_{n}}{P_{n}(\Omega)}\right) \leq n\left[\int_{\Omega} \phi(X) d Q-\phi\left(\int_{\Omega} X d Q\right)\right]=0 .
$$

Thus, for every $n$ we have

$$
\int_{\Omega} \phi(X) \frac{d P_{n}}{P_{n}(\Omega)}=\phi\left(\int_{\Omega} X \frac{d P_{n}}{P_{n}(\Omega)}\right) .
$$

Since all the limits involved exist, letting $n \rightarrow \infty$ and using the continuity of $\phi$ in the interior of its domain $I$, we conclude that

$$
\int_{\Omega} \phi(X) d P=\phi\left(\int_{\Omega} X d P\right)
$$

(this is stated below as a corollary, as it seems to be of independent interest). Note that the interval $I$ might contain one (or both) of its endpoints, say it contains the endpoint $a$, and $\phi$ might be discontinuous there. However, if $\int_{\Omega} X d P=a$, since the range of $X$ is contained in $I$, we have $X=a P$-a.e., and thus (13) also holds in this case.

So far, we know that if $\int_{\Omega} \phi(X) d P<\infty$ and $\int_{\Omega} \phi(X) d Q<\infty$, then inequality (41) holds, regardless of whether or not $\frac{d P}{d Q}$ is essentially bounded, and additionally, that if $\int_{\Omega} \phi(X) d Q=\phi\left(\int_{\Omega} X d Q\right)$, then $\int_{\Omega} \phi(X) d P=\phi\left(\int_{\Omega} X d P\right)$. Furthermore, it is clear that if $\int_{\Omega} \phi(X) d P=\infty$ and $\left\|\frac{d P}{d Q}\right\|_{\infty}<\infty$, we must have $\int_{\Omega} \phi(X) d Q=$ $\infty$, so all we need to show is that $\left\|\frac{d P}{d Q}\right\|_{\infty}=\infty$ whenever $\int_{\Omega} \phi(X) d P=\infty$ and $\int_{\Omega} \phi(X) d Q<\infty$. This latter inequality, together with $\int_{\Omega} \phi(X) d Q \geq \phi\left(\int_{\Omega} X d Q\right)>$ $-\infty$, entails that both the positive and negative parts of $\phi(X)$ are $Q$-integrable. 
Suppose, towards a contradiction, that $\left\|\frac{d P}{d Q}\right\|_{\infty}<\infty$. Then

$$
\int_{\Omega} \phi(X) d P \leq \int_{\Omega}\left|\phi(X) \frac{d P}{d Q}\right| d Q \leq\left\|\frac{d P}{d Q}\right\|_{\infty} \int_{\Omega}|\phi(X)| d Q<\infty .
$$

In view of the fact that inequality (4) holds under no restrictions, the first equality case, where both sides take the value $\infty$, follows immediately.

Next, suppose that $\phi$ is strictly convex. By the equality case of Jensen's inequality the right hand side of (4) is zero (hence, so is the left hand side) if and only if $X$ is constant $Q$-a.e.; thus, statement 2 of the theorem is true.

Finally, suppose there exists a constant $a \in(0, \infty)$ such that

$$
a=\int_{\Omega} \phi(X) d P-\phi\left(\int_{\Omega} X d P\right)=\left\|\frac{d P}{d Q}\right\|_{\infty}\left(\int_{\Omega} \phi(X) d Q-\phi\left(\int_{\Omega} X d Q\right)\right) .
$$

Then a) is immediate; using (4) we conclude that $\int_{\Omega} \phi(X) d P$ is also finite, so equality on (4) is equivalent to having equality on (7), which in turn is equivalent to $\phi\left(\int_{\tilde{\Omega}} \tilde{X} d \tilde{P}\right)=\int_{\tilde{\Omega}} \phi(\tilde{X}) d \tilde{P}$. Now $X$ is not constant $Q$-a.e. on $\Omega$ (by the equality case in Jensen's inequality, otherwise $a$ would be zero), but $\tilde{X} i s$ constant $\tilde{P}$-a.e. by the equality case in Jensen's inequality; call this constant c. Since by Lemma 2.7 $\tilde{P}$ and $Q$ are mutually absolutely continuous over the set $\Omega \backslash A$, statement b) follows.

To see why c) holds, note first that if $Q(A)=0$, then by b) $X \equiv c Q$-a.e. and we are in the case $0=0$. However, $a>0$, so $Q(A)>0$, and hence $P(A)>0$ by Lemma 2.6. Since $P \neq Q$, we also have $\tilde{P}(\Omega \backslash A)>0$. Using $\tilde{X} \equiv c \tilde{P}$-a.e. together with (8)-(9), we see that for $\tilde{P}$-a.e. (or $Q$-a.e.) $w \in \Omega \backslash A$,

$$
\int_{\Omega} X d Q=\int_{\tilde{\Omega}} \tilde{X} d \tilde{P}=c=X(w)=\tilde{X}(y)=\int_{\Omega} X d P .
$$

Since $X=c$ a.e. (with respect to all measures under consideration) on $\Omega \backslash A$,

$\mathrm{SO}$

$$
\int_{\Omega \backslash A} X d Q+\int_{A} X d Q=c(1-Q(A))+\int_{A} X d Q=\int_{\Omega} X d Q=c,
$$

$$
c=\frac{1}{Q(A)} \int_{A} X d Q
$$

The fact that

$$
c=\frac{1}{P(A)} \int_{A} X d P
$$

is obtained in the same way. Alternatively, since $\int_{\Omega} X d Q=\int_{\Omega} X d P$ and $X=c$ a.e. on $X \backslash A$, we see that (16) holds if and only if (17) holds.

Suppose now that a), b) and c) hold. Using a) and b) we conclude that the right hand side of (4) is neither zero nor infinity. Since all terms involved are finite, equality on (4) is equivalent to $\phi\left(\int_{\tilde{\Omega}} \tilde{X} d \tilde{P}\right)=\int_{\tilde{\Omega}} \phi(\tilde{X}) d \tilde{P}$, which holds if $\tilde{X}$ is $\tilde{P}$-a.e. constant on $\tilde{\Omega}$. We prove this next. First, $\tilde{X} \equiv c \tilde{P}$-a.e. on $\Omega \backslash A$ by b) and Lemma 2.7 while by definition and by c), $\tilde{X}(y)=\int_{\Omega} X d P=c$. Since $\tilde{P}(A)=0$ by Lemma 2.7 the result follows.

The next corollary was obtained as a step in the preceding proof, and of course, it also follows directly from the statement of Theorem 2.1. 
Corollary 2.8. Let $(\Omega, \mathcal{A}, Q)$ be a probability space, let $X: \Omega \rightarrow \mathbb{R}$ be integrable, and let $\phi$ be real valued and convex in some interval containing the range of $f$. If $\phi\left(\int_{\Omega} X d Q\right)=\int_{\Omega} \phi(X) d Q$, then for every probablity $P \ll Q$ such that $X \in L^{1}(P)$, we have $\phi\left(\int_{\Omega} X d P\right)=\int_{\Omega} \phi(X) d P$.

Proof. By (4), since $\int_{\Omega} \phi(X) d P-\phi\left(\int_{\Omega} X d P\right) \geq 0$.

We next state the version of Theorem 2.1 (where for simplicity we omit the analogous equality conditions) that provides a lower bound (instead of an upper bound) using the essential infimum of the Radon-Nykodim derivative (instead of the essential supremum). Recall that if $P$ and $Q$ are mutually absolutely continuous and $h$ is any representative of $\frac{d P}{d Q}$, then $1 / h$ is a representative of $\frac{d Q}{d P}$.

Corollary 2.9. Let $(\Omega, \mathcal{A})$ be a measurable space, let $P$ and $Q$ be probability measures defined on $(\Omega, \mathcal{A})$ such that $P \ll Q$, let $X: \Omega \rightarrow \mathbb{R}$ be integrable both with respect to $P$ and $Q$, and let the real-valued function $\phi$ be convex in some interval containing the range of $X$. Then

$$
\left(\operatorname{ess} \inf \frac{d P}{d Q}\right)\left(\int_{\Omega} \phi(X) d Q-\phi\left(\int_{\Omega} X d Q\right)\right) \leq \int_{\Omega} \phi(X) d P-\phi\left(\int_{\Omega} X d P\right) .
$$

Proof. If ess $\inf \frac{d P}{d Q}=0$, then inequality (18) reduces to the usual Jensen inequality, so only when ess $\inf \frac{d P}{d Q}>0$ does (18) say something new. However, in this case we have $Q \ll P$, with $\frac{d Q}{d P}=\frac{1}{d P / d Q}$ a bounded function, since $\left\|\frac{d Q}{d P}\right\|_{\infty}=\frac{1}{\text { ess inf } \frac{d P}{d Q}}<\infty$. Now inequality (18) follows from (4): Multiply both sides of (18) by $\left\|\frac{d Q}{d P}\right\|_{\infty}$ and note that this is just (4) with the roles of $P$ and $Q$ interchanged.

Since in the non-trivial case ess inf $\frac{d P}{d Q}>0$ the preceding corollary reduces to Theorem 2.1, the corresponding equality conditions follow automatically.

If $\phi$ is concave, then applying Theorem 2.1 and Corollary 2.9 to $-\phi$ we obtain the following.

Corollary 2.10. Let $(\Omega, \mathcal{A})$ be a measurable space, let $P$ and $Q$ be probability measures defined on $(\Omega, \mathcal{A})$ such that $P \ll Q$, let $X: \Omega \rightarrow \mathbb{R}$ be integrable both with respect to $P$ and $Q$, and let the real-valued function $\phi$ be concave in some interval containing the range of $X$. Then

$$
\begin{gathered}
\left(\operatorname{essinf} \frac{d P}{d Q}\right)\left(\phi\left(\int_{\Omega} X d Q\right)-\int_{\Omega} \phi(X) d Q\right) \\
\leq \phi\left(\int_{\Omega} X d P\right)-\int_{\Omega} \phi(X) d P \leq\left\|\frac{d P}{d Q}\right\|_{\infty}\left(\phi\left(\int_{\Omega} X d Q\right)-\int_{\Omega} \phi(X) d Q\right) .
\end{gathered}
$$

Next we state the corresponding refinement of the measure-theoretic version of the arithmetic-geometric mean inequality $\exp \int_{\Omega} \log (X) d P \leq \int_{\Omega} X d P$, thereby generalizing [Al, Theorem 2.1]. 
Corollary 2.11. Let $(\Omega, \mathcal{A})$ be a measurable space, let $P \ll Q$ be probability measures defined on $(\Omega, \mathcal{A})$, and let $X: \Omega \rightarrow[0, \infty)$ be such that $\log (X)$ is integrable both with respect to $P$ and $Q$. Then

$$
\begin{gathered}
\left(\operatorname{ess} i n f \frac{d P}{d Q}\right)\left(\int_{\Omega} X d Q-\exp \int_{\Omega} \log (X) d Q\right) \\
(22) \leq \int_{\Omega} X d P-\exp \int_{\Omega} \log (X) d P \leq\left\|\frac{d P}{d Q}\right\|_{\infty}\left(\int_{\Omega} X d Q-\exp \int_{\Omega} \log (X) d Q\right) .
\end{gathered}
$$

Let us finish by saying that the reader interested in applications of the DragomirJensen inequality to information inequalities, can find some such applications (for the discrete case) in Dragomir's original paper Dra].

\section{REFERENCES}

[Al] Aldaz, J. M. Comparison of differences between arithmetic and geometric means. Tamkang J. of Math., to appear. Available at the mathematics arXiv.

[Dra] Dragomir, Sever S. Bounds for the normalised Jensen functional. Bull. Austral. Math. Soc. 74 (2006), no. 3, 471-478. MR2273755 (2007f:26021)

Departamento de Matemáticas, Universidad Autónoma de Madrid, Cantoblanco 28049, MAdrid, Spain

E-mail address: jesus.munarriz@uam.es 